Research Article

\title{
Effect of Viscosity and Density of Substance on Dielectric Properties of Medicinal Compounds in Solution
}

\author{
Jinan Fadhil Mahdi ${ }^{1}$, Ansari Abdul Jaleel Abdul Haque², Mazahar Ahmed Nazeeruddin Farooqui ${ }^{3}$, \\ Yusuf Hanif Shaikh ${ }^{4}$ \\ ${ }^{1}$ College of Electrical Engineering Techniques, Middle Technical University, Ministry of Higher Education and Scientific Research, \\ Iraq. \\ ${ }^{2}$ Department of Physics and Electronics, Maulana Azad College of Arts, Science and Commerce, Aurangabad, Maharashtra, India. \\ ${ }^{3}$ Department of Chemistry, Maulana Azad College of Arts, Science and Commerce, Aurangabad, Maharashtra, India. \\ ${ }^{4}$ Department ofPhysics and Electronics, Shivaji College, Kannad, Aurangabad, Maharashtra, India. \\ Corresponding author. E-mail: Jinan.f@mtu.edu.iq; jinanf06@gmail.com
}

Received: May 17, 2020; Accepted: Sep. II, 2020; Published: Dec. 3, 2020

Citation: Jinan Fadhil Mahdi, Ansari Abdul Jaleel Abdul Haque, Mazahar Ahmed Nazeeruddin Farooqui, and Yusuf Hanif Shaikh, Effect of Viscosity and Density of Substance on Dielectric Properties of Medicinal Compounds in Solution. Nano Biomed. Eng., 2020, I2(4): 35 I-357.

DOI: 10.5101/nbe.v12i4.p351-357.

\begin{abstract}
Complex permittivity in terms of dielectric constant $\left(\varepsilon^{\prime}\right)$ and dielectric loss $\left(\varepsilon^{\prime \prime}\right)$ of medicinal compound paracetamol in dimethyl sulfoxide (DMSO) solvent was determined with different weight fractions at microwave frequency $9.85 \mathrm{GHz}$ at constant temperature $27^{\circ} \mathrm{C}$. The information on dielectric properties is related to the properties of the substance for preparation of granules while making dosage forms like tablets and capsules. Refractive index $(\mathrm{nD})$, dielectricconstant $\left(\varepsilon^{\prime}\right)$ and dielectric loss $\left(\varepsilon^{\prime \prime}\right)$ were used for the measurement of relaxation time $(\tau)$. The viscosity $(\eta)$ and density of paracetamol in DMSO were determined using a conventional Ostwald's viscometer and specific gravity bottle respectively. The aim of the present work was to study the dielectric properties of the drugs and attempted to determine their correlations with density and viscosity to understand the behavior of the dielectric properties under different conditions like a concentration in solution. It was observed that there was a strong correlation of dielectric properties like dielectric constant, dielectric loss and dielectric relaxation time with viscosity and density. The absolute values of correlation coefficient $(\mathrm{R})$ were in the range of 0.946 to 0.987 for paracetamol-DMSO system, indicating a strong correlation.
\end{abstract}

Keywords: Dielectric constant, Microwave bench, Magnetic stirrer, Ostwald's viscometer, Specific gravity bottle, Relaxation time, Debye model

\section{Introduction}

About dielectric properties, the electromagnetic properties of materials of interest to scientists and engineers include the dielectric constant, relaxation time and dielectric loss. Electromagnetic wave has worldwide application for medical therapy and diagnostics in electromagnetic based medical technologies $[1,2]$. For dielectric materials, both for loss and lossless, the most identifying parameter is the dielectric constant or permittivity. Dielectric properties are intrinsic characteristics of the materials explaining the behavior and degree of the wave-matter interaction when exposed to microwave field. These properties 
are very important in microwave heating, microwave sensing, process design and application [3-7].

Apart from this, many researchers have used dielectric properties to measure moisture content of material and agro-food. The measurement of dielectric properties of liquid materials at microwave frequencies can be used to determine some electrical properties and evaluation of biological effects in biological molecules $[8,9]$. Dielectric properties are utmost useful property as rapid and non-destructive method. So the microwave dielectric properties can be used for set up to measure drug dosage in solvent [10]. Dielectric polarization and dielectric relaxation can be used for systematic explanation in inner polarization mechanism of molecules. Due to limitation of current research and difficulties of polarization process many unclear issue can be solve by systematic approach of said properties [11].

If the polar solute molecules are spherical, and large in comparison with the solvent molecules, then the orientation relaxation of the solute molecules can usefully be described using Debye's model. In this model, the bipolar solute molecules are considered as spheres whose rotation was opposed by the viscosity of the surrounding solvent medium [12]. The relaxation time $\tau$ can be determined by

$\tau=\left(4 \pi \eta \mathrm{a}^{2}\right) /(\mathrm{KT})$.

Here $\tau$ is relaxation time, $\eta$ is the viscosity of liquid, and $\mathrm{a}$ is radius of spherical solute molecule. According to Equation (1), relaxation time varies with variation of viscosity for same solute-solvent system at constant temperature. Then,

$\tau=\mathrm{A} \eta$,

where $\mathrm{A}=4 \pi \mathrm{a}^{3} / \mathrm{KT}, \mathrm{K}$ is the Boltzmann constant, and $\mathrm{T}$ is the absolute temperature. The relaxation time of liquid can be determined by using dielectric properties is given below in Equation (3).

$\tau=\varepsilon^{\prime \prime} /\left[\omega\left(\varepsilon^{\prime}-\mathrm{n}_{\mathrm{D}}^{2}\right)\right]$

where $\varepsilon^{\prime}$ and $\varepsilon^{\prime \prime}$ are dielectric constant and dielectric loss respectively, $\mathrm{n}_{\mathrm{D}}$ is the refractive index, and $\omega$ is the angular velocity. The relaxation time of biological molecule is related to the size of molecule in liquid, mobility of molecules etc. Small sizes of molecules indicate lower relaxation time; an increase in size of molecule increases the relaxation time [13, 14]. Similarly, density was directly affected on mobility and viscosity of molecules. Study of dielectric properties of medicinal substances and drug has applications in Medicine, Pharmacy and drug administration as these properties help designing granulation process for oral dosage forms like tablets and capsules. Dimethyl sulfoxide (DMSO) is an important polar aprotic solvent that dissolves both polar and nonpolar compounds. Therefore, it is used in extraction and coating of such compounds. As it is used in the industry, related information is valuable to industry.

\section{Experimental}

The two medicinal drugs paracetamol and Dimethyl sulfoxide (SIGMA ALDRICH Co. USA) were purchased (Doodle Chem. Pvt. Ltd. India) and used as the starting materials. Dielectric properties of these medicinal compounds were measured at $9.85 \mathrm{GHz}$ using microwave X-band in solution. The present work relates to the study of permittivity of some medicinal powder in solvent. The dielectric properties have been measured at $9.85 \mathrm{GHz}$ for mode TE10 and temperature of $27{ }^{\circ} \mathrm{C}$ by using waveguide plunger technique. All properties were measured by using Heston and Smyth method [16-22] at room temperature $27^{\circ} \mathrm{C}$. Dielectric measurements of the powders and all mixtures were carried out in the DMSO and changing the wt fraction of powder in the range $0-1.0 \mathrm{~kg} / \mathrm{kg}(0.1$ or $0.2 \mathrm{~kg} / \mathrm{kg}$ intervals).

For each experiment, the powder is dissolved in solvent using Magnetic Stirrer and used for further analysis. Dielectric measurements were made at standard frequency of $9.85 \mathrm{GHz}$ for investigation with various Powder- Solvent concentrations. Each experiment was performed in triplicate.

The incident and reflected intensity of microwave was used for calculation of VSWR using the relation [23]:

$V S W R=\mathrm{S}=\frac{\left[{ }^{\left.I_{f}+I_{r}+2\left(I_{r} I_{f}\right)^{\frac{1}{2}}\right]}\right.}{\left(I_{f}-I_{r}\right)}$.

Here $I_{f}$ is the forward current and $I r$ is the reverse current due to reflected wave.

The block diagram of the experimental setup used for the study of the dielectric properties is shown in Fig. 1.

From Heston Smyth Method [23], dielectric constant $\varepsilon^{\prime}$ and dielectric loss $\varepsilon^{\prime \prime}$ can be calculated by 


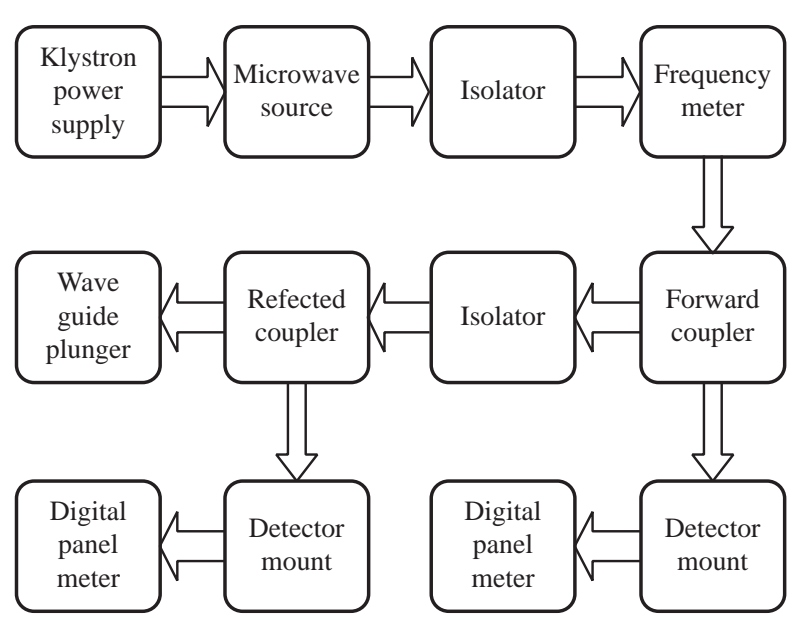

Fig. 1 The block diagram of microwave slotted section set-up.

$\varepsilon^{\prime}=\left[\lambda_{0} / \lambda_{\mathrm{c}}\right]^{2}+\left[\lambda_{0} / \lambda_{\mathrm{d}}\right]^{2}$,

and

$\varepsilon^{\prime \prime}=2 / \pi\left(\lambda_{0} / \lambda_{\mathrm{d}}\right)^{2}\left(\lambda_{\mathrm{g}} / \lambda_{\mathrm{d}}\right)\left(\mathrm{d} \rho_{\text {mean }} / \mathrm{dn}\right)$,

where $\lambda_{0}$ is the free space wavelength, $\lambda_{\mathrm{d}}$ is wavelength in dielectric, $\lambda_{c}$ is cut off wavelength $=2 a$. Here ' $a$ ' is the width of the cell and $\lambda_{g}$ is guide wavelength. To determine the dielectric loss, a graph is plotted between several mean values of $\rho$ versus $n$. The slope of this graph gives the value of

$\mathrm{d} \rho_{\text {mean }} / \mathrm{dn}$.

Co-relation between dielectric and physical properties of liquid has its own importance for understanding the physical and electrical behavior of liquid. Relaxation time depends on some physical properties i.e. density, viscosity and refractive index etc. Refractive index of liquid is used for measurement of dielectric constant of liquid or solid; we know that the relationship between relaxation time and refractive index is given by Equation (3).

If the polar solute molecules are spherical, and large by comparison with the solvent molecules, then the orientation relaxation of the solute molecules can usefully be described using Debye's model. In this model, the bipolar solute molecules are considered as spheres whose rotation is opposed by the viscosity of the surrounding solvent medium [14].

$\tau=\left(4 \pi \eta \mathrm{a}^{2}\right) /(\mathrm{KT})$.

Here again, $\tau$ is relaxation time of mixture, $\eta$ is a viscosity of liquid, and a is radius of spherical solute. According to Equation (1), relaxation time varies with variation of viscosity for same solute-solvent system at constant temperature.

Relaxation time of biological molecule is related to the size of molecule in liquid, mobility of molecules etc. Small sizes of molecule indicate low viscosity as well as lower relaxation time, increase in size of molecule increases the relaxation time [24, 25]. Similarly, density of liquid also depends upon viscosity and mobility of molecules, density is directly affected on mobility and viscosity of molecules.

Density of liquid was measured using water as a reference and is given by

$\rho_{\mathrm{s}}=\left(\mathrm{m}_{\mathrm{s}} / \mathrm{m}_{\mathrm{w}}\right) \rho_{\mathrm{w}}$.

Here, $\rho_{\mathrm{S}}$ is the relative density of sample, $\rho_{\mathrm{W}}$ is the density of water, $\mathrm{m}_{\mathrm{S}}$ is the mass of sample, and $\mathrm{m}_{\mathrm{W}}$ is the mass of water.

Viscosity can be measured by using Ostwald's viscometer by using the given formula

$\eta_{2}=\eta_{1}\left(\rho_{1} t_{1} / \rho_{2} t_{2}\right)$,

where $\eta_{1}$ is a viscosity of known liquid (water), $\eta_{2}$ is a viscosity of unknown liquid, $\rho_{1}$ is a density of known liquid (water), and $\rho_{2}$ is a density of unknown liquid.

Co-efficient of correlation shows the relation between two properties, which can be determined by the relation $[26,27]$,

$$
\operatorname{Correl}(X, Y)=\frac{\Sigma(x-\bar{x})(y-\bar{y})}{\sqrt{\Sigma(x-\bar{x})^{2} \Sigma(y-\bar{y})^{2}}},
$$

where $\mathrm{x}$ and $\mathrm{y}$ are the sample means average (array 1) and average (array 2).

Co-relation shows the relation between two properties, positive co-relation indicates the relation between properties present, a value close to +1 indicates a strong positive relationship. Zero value of correlation of two properties shows there is no relation between these properties. Negative values of correlation coefficient indicates that there is a negative relationship indicating that increase in one results in decrease of the other property, a lower value indicates weak or poor correlation and a higher value close to +1 indicates a strong positive relation.

\section{Results and Discussion}

Co-relation between dielectric and physical properties of liquid has its own importance for understanding the physical and electrical behavior of liquid. Relaxation time depends on some physical properties i.e. density, viscosity and refractive index etc. Refractive index of liquid is used for measurement 
Table 1 The physical and dielectric properties of paracetamol in DMSO

\begin{tabular}{|c|c|c|c|c|c|c|}
\hline \multirow{2}{*}{ Ratio of concentration in $\mathrm{kg} / \mathrm{kg}$} & \multicolumn{6}{|c|}{ Paracetamol } \\
\hline & Refractive index & Density in $\mathrm{gm} / \mathrm{cm}^{3}$ & Viscosity in $\mathrm{m}$ poise & $\varepsilon^{\prime}$ & $\varepsilon^{\prime \prime}$ & Relaxation time in ps \\
\hline $1: 0$ & 1.487 & 1.110 & 19.000 & 31.3900 & 22.1100 & 12.533 \\
\hline $1: 0.1$ & 1.488 & 1.115 & 79.650 & 25.1961 & 18.8975 & 12.767 \\
\hline $1: 0.2$ & 1.488 & 1.116 & 140.300 & 20.2244 & 16.5800 & 13.107 \\
\hline 1:0.3 & 1.490 & 1.124 & 200.950 & 16.2337 & 14.8425 & 13.447 \\
\hline $1: 0.4$ & 1.492 & 1.128 & 261.600 & 13.0305 & 13.4988 & 13.715 \\
\hline $1: 0.5$ & 1.500 & 1.133 & 322.250 & 10.4593 & 12.4331 & 13.949 \\
\hline $1: 0.6$ & 1.502 & 1.135 & 362.900 & 8.3954 & 11.5699 & 14.184 \\
\hline $1: 0.7$ & 1.504 & 1.143 & 413.550 & 6.7389 & 10.8581 & 14.220 \\
\hline $1: 0.8$ & 1.505 & 1.147 & 504.200 & 5.4091 & 10.2623 & 14.345 \\
\hline $1: 0.9$ & 1.511 & 1.152 & 540.850 & 4.3418 & 9.7569 & 14.586 \\
\hline $1: 1$ & 1.518 & 1.157 & 582.500 & 3.4851 & 8.3668 & 15.000 \\
\hline
\end{tabular}

of dielectric constant of liquid or solid. Dielectric relaxation time of material is an important parameter to understand the behavior of molecule and it also calculated by physical method which directly depends on viscosity.

Dielectric and physical properties of paracetamol in DMSO were measured for determination of the correlation between them. Dielectric properties like dielectric constant, dielectric loss and relaxation time were measured at $9.85 \mathrm{GHz}$ and refractive index, viscosity and density are also determined at constant temperature of $27^{\circ} \mathrm{C}$ and tabulated in Table 1 .

Fig. 2 and 3 show the graphical presentation of variation of relaxation time with viscosity and density at different weight fraction of paracetamol in DMSO. It is clearly seen that relaxation time increases with an increase in viscosity, viscosity of molecules depends

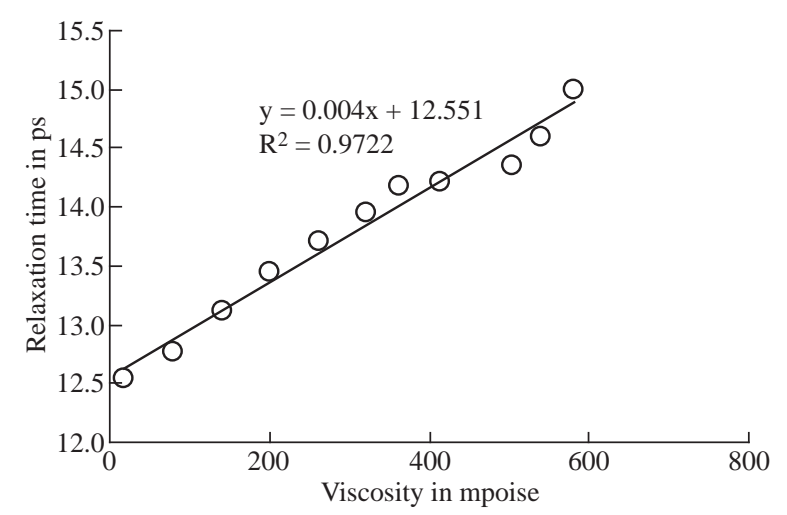

Fig. 2 The viscosity and relaxation time of paracetamol in DMSO.

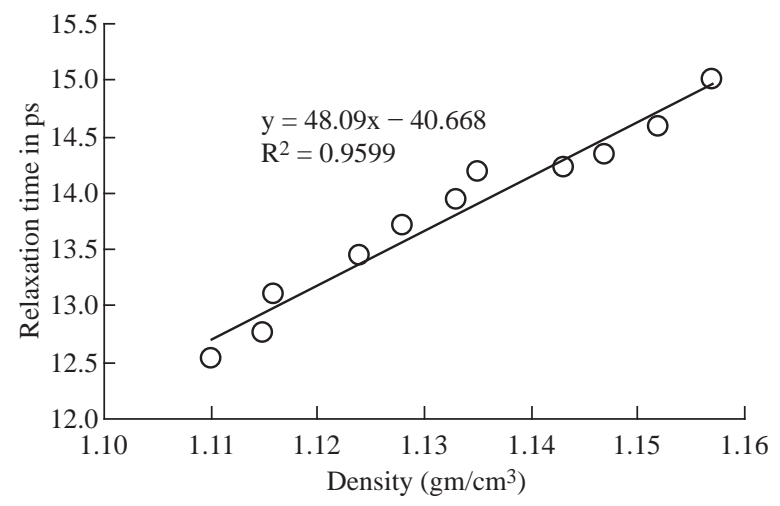

Fig. 3 The density and relaxation time of paracetamol in DMSO.

on the size of molecules in Fig. 2. This is obeying the Debye theory of relaxation. Relaxation time increase increases with increasing the viscosity in linear trend, the trend shows the best fitting line $\mathrm{R}^{2}$ value is 0.9722. Fig. 3 shows the graphical representation of variation of density and relaxation time with variation of wt fraction of paracetamol in DMSO. The purpose of the graph of relaxation time and density with wt fraction of paracetamol in DMSO is to compare both said properties and determination of correlation between them. It is seen from the Fig. 3 that both the properties increase with increase in weight fraction of paracetamol in DMSO however the trends are a bit different. The correlation between these two physical properties was determined using Equation (8) and presented in Table 2.

It is clearly seen that the relaxation time increase 
Table 2 Correlation between physical and dielectric properties of paracetamol-DMSO system

\begin{tabular}{ccc}
\hline & Paracetamol-DMSO system & \\
\hline Parameters & Correlation & Inference \\
\hline Density and dielectric constant & -0.9468 & Strong negative correlation \\
Viscosity and dielectric constant & -0.9630 & Strong negative correlation \\
Density and dielectric loss & -0.9496 & Strong negative correlation \\
Viscosity and dielectric loss & -0.9644 & Strong negative correlation \\
Density and relaxation time & 0.9776 & Strong positive correlation \\
Viscosity and relaxation time & 0.9625 & Strong positive correlation
\end{tabular}

with increasing viscosity of paracetamol-DMSO system at different wt fraction of paracetamol in DMSO. The trend of graph is linear with the best fitting line $\mathrm{R}^{2}$ value, i.e 0.9265 . This incorporates the concept of visco-elastic relaxation, which is generally true for dielectric systems obeying Debye theory of relaxation.

Fig. 4 and 5 show the variation of dielectric constant with variation of density and viscosity respectively. It is seen that dielectric constant having relation with density as well as viscosity with in linear trend and best

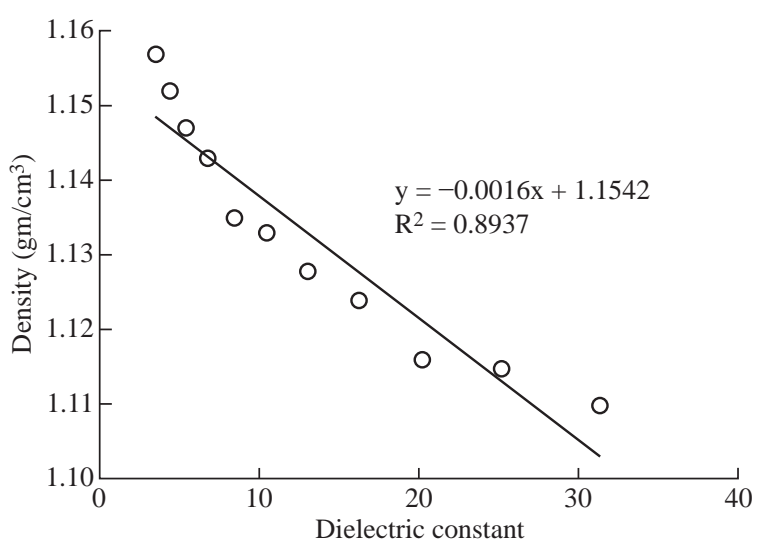

Fig. 4 The density and Dielectric constant of paracetamol in DMSO.

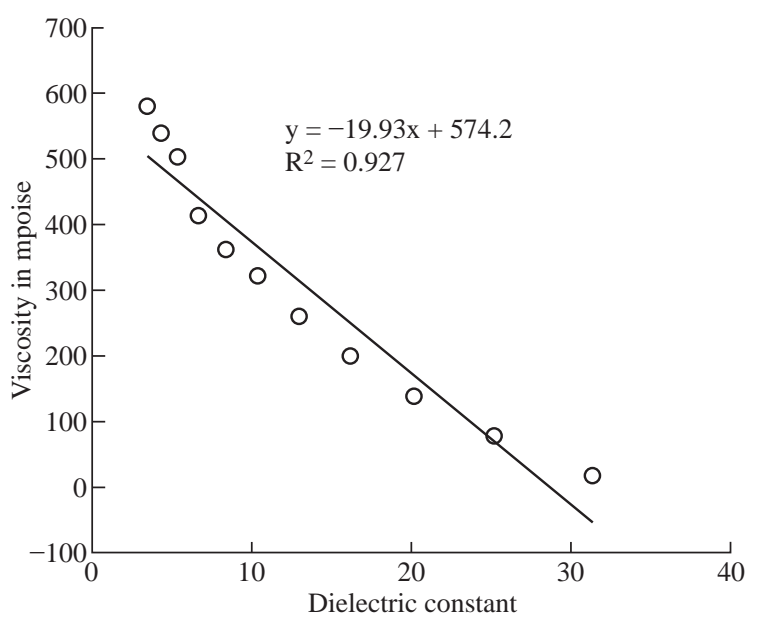

Fig. 5 The viscosity and Dielectric constant of paracetamol in DMSO. fitting line $\mathrm{R}^{2}$ values are 0.893 and 0.927 , respectively.

Similarly, correlation between dielectric loss with physical properties (density and viscosity) are attempted and presented in Fig. 6 and 7. It is clearly seen that dielectric loss decreases with increase in density and viscosity a linear manner with 0.899 and 0.930 best fitting line $\mathrm{R}^{2}$ values respectively.

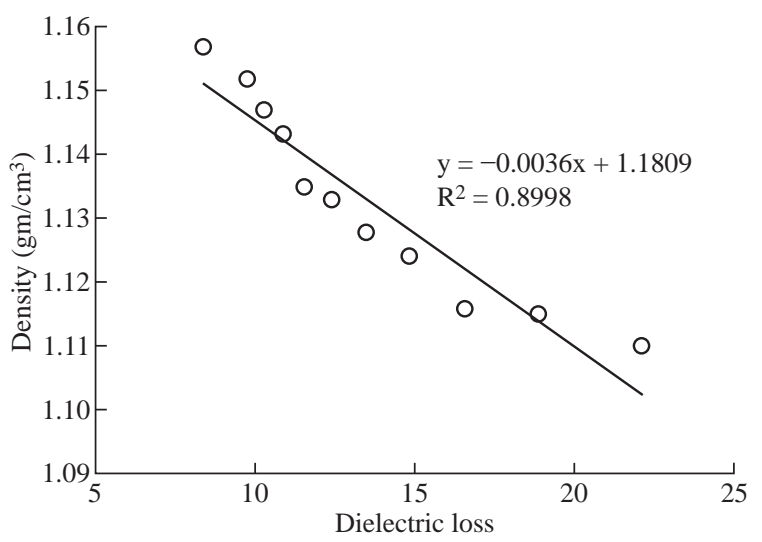

Fig. 6 The density and Dielectric constant of paracetamol in DMSO.

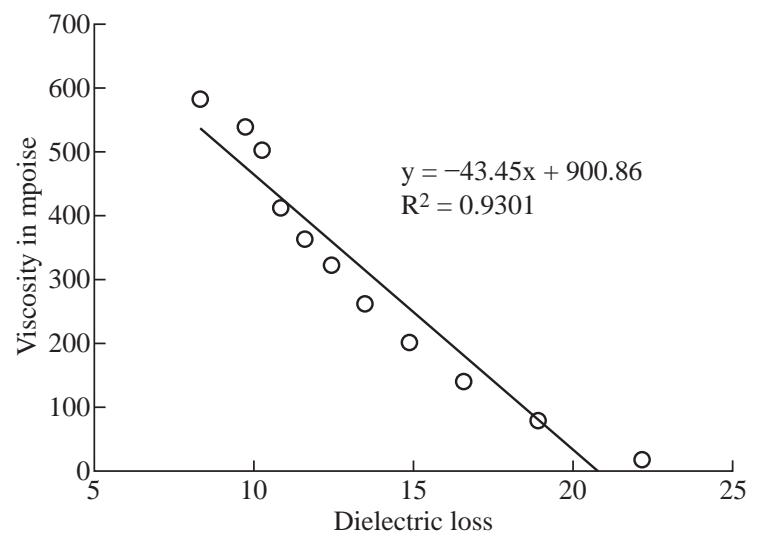

Fig. 7 The viscosity and Dielectric loss of paracetamol in DMSO.

Dielectric properties of super-cooled methanol were carried out by Li et al. [28] at different temperatures and frequencies for investigation of effect of temperatures and frequencies on Debye relaxation time. 
They are reported that the Debye relaxation time gives the knowledge of ideal mixture law, which is helpful to evaluate the dynamics in depth. In addition, broadened Debye relaxation is expected in the dielectric spectra of the methanol, and this is quite different from earlier observation of the Debye liquids.

Comparative studies of dielectric properties of ferrous gluconate-milk and ferrous chloride-milk were carried out for estimation of microwave absorption, more or less absorption of microwave leads to more or less heat generation [29]. Conversion of heat from the microwave power is purely depends on the coefficient of microwave absorption which is explained in terms of dielectric loss. Relaxation process of molecules affected by the variations of density, rotation motion of molecules and inter and intramoleculer forces of bound water and corporate the established of free water respectively [30].

Relaxation time of biological molecule in liquid depends on the mobility of molecules, size of molecules, viscosity of liquid, density and temperature. The value of relaxation time of liquid increases with increase in density, viscosity and size of molecule. Viscosity opposes the bipolar rotation of molecule; it increases the time of reorientation of molecule resulting in an increasing of the relaxation time. Variation of density and viscosity are always in direct correlation, both properties depend on each other [31]. The correlation between viscosity and relaxation time and density and relaxation time are found to be positive for paracetamol- DMSO system. The value of correlation between density and relaxation time is 0.9776; it indicates that the strong positive correlation present between them. Viscosity and relaxation time directly depend to each other. The correlation between viscosity and relaxation time obtained is 0.9625 . It indicates that strong positive correlation is present between viscosity and relaxation time of paracetamolDMSO system.

Correlation between physical and dielectric properties of paracetamol in DMSO was found and is tabulated in Table 2.

The absolute values of correlation coefficient $\mathrm{R}$ are in the range of 0.946 to 0.977 for paracetamol DMSO system indicating a strong correlation, first four properties exhibits a negative correlation indicating that an increase in one property corresponds to a decrease in the other property. The last two properties exhibit a positive correlation coefficient indicating that an increase in one property corresponds to an increase in the other.

Dielectric constant, density and viscosity vary with variation of wt fraction of solute in solvent. The correlation of dielectric constant with density and viscosity are obtained -0.9630 and -0.9496 for paracetamol in DMSO. This negative correlation shows there is strong negative relation present between dielectric constant and density and dielectric constant and viscosity. However, the dielectric constant, density and viscosity vary in same manner with variation of $\mathrm{wt}$ fraction of solute in solvent.

Similarly, the correlation of dielectric loss with density and viscosity are also negative. It shows that there is strong negative correlation present between dielectric loss and density and dielectric loss and viscosity as is shown in Table 2 . The negative correlation implies that an increase in one parameter corresponds to a decrease in the other. The values of correlation obtain between density and dielectric loss is -0.9496 for paracetamol-DMSO system. Values of correlation coefficient for paracetamol- DMSO obtained between viscosity and dielectric loss are -0.9644 . It indicates that strong negative correlation is present between these properties.

\section{Conclusions}

Correlation between physical and dielectric properties was attempted to understand the behavior of the dielectric properties under different conditions like concentration in solution. It was observed that there was a strong correlation between viscosity and density with dielectric constant, dielectric loss and relaxation time. Debye relaxation of paracetamol-DMSO mixture gave the information of molecular rotation of solute molecule in mixture. Relaxation time increased with increase in viscosity and density and obeyed the Debye relaxation model.

\section{Conflict of Interests}

The author hereby declared no conflict of interest regarding the manuscript and experimentation done.

\section{References}

[1] A. La Gioia, E. Porter, I. Merunka, et al., Open-Ended Coaxial Probe Technique for Dielectric Measurement of 
Biological Tissues: Challenges and Common Practices. Journal of Diagnostics, 2018, 8(40): 3-38.

[2] D.E. Khaled, N. Novas, J.A. Gazquez, et al., Dielectric and Bioimpedance Research Studies: A Scientometric Approach Using the Scopus Database. Journal of Mpdi, 2018, 6(6).

[3] F Peyre, A. Datta, and C Seyler, Influence of the Dielectric Property on Microwave Oven Heating Patterns: Application to Food Materials. The Journal of Microwave Power and Electromagnetic Energy, 1997, 32(1): 3-15.

[4] T.R.A. Magee, Moisture and temperature dependence of the dielectric properties of pharmaceutical powders. $J$ Therm Anal Calorim, 2013, 1(11): 2157-2164.

[5] C.M. McLoughlin, W.A.M. McMinn, and T.R.A. Magee, Physical and Dielectric Properties of Pharmaceutical Powders. Powder Technology, 2003, 13(4): 40-51.

[6] R.E. Mudgett, Microwave properties and heating characteristics of foods. Food Technology, 1986, 40(3): 84-93.

[7] A.S. Mujumdar, Handbook of industrial Drying Vol-1, (Second Edition). New York: Marcel Dekker, 1995: 345372.

[8] W. Kuang, S.O. Nelson, Low-frequency dielectric properties of biological tissues. ASAE, 1998, 41(1): 173184.

[9] D. Faktorova, Complex Permittivity of Biological Materials Measurement at Microwave Frequencies. Measurement Science Review, 2007, 7(2): 12-15.

[10] R.V. Decareau, Microwaves in the food processing industry. New York: Academic Press, 1985: 148-164.

[11] M. Fareq, Dielectric Spectroscopy of Pharmaceutical Drug (paracetamol) Dosage in Water. Proceedings of 2013 IEEE International RF and Microwave Conference (RFM2013). Penang, Malaysia, Dec. 9-11, 2013: 218225.

[12] B. Quan, X. Liang, G. Ji, et al., Dielectric polarization in electromagnetic wave absorption: Review and perspective. Journal of Alloys and Compounds, 2017, 72(8): 1065-1075.

[13] G.M. Walker, T.R.A. Magee, C.R. Holland, et al., Caking processes in granular NPK fertilizer. Ind Eng Chem Res., 1998, 37(2): 435-438.

[14] P. Debye, Dielectric constant. Energy absorption in dielectrics with polar molecules. Trans. For. Soc., 1934, 30: 679.

[15] R. Von Hippel, Dielectric and waves. The M.I.T. Press, 1955: 214-228.

[16] W. Daseler, H.J. Steinhoff, and A. Redhardt, A new method for the determination of the permittivity of small samples in the microwave range and its application to hemoglobin single crystals. Journal of Biochemical and Biophysical Methods, 1991, 22(1): 69-82.

[17] Y. Wang, M.N. Afsar, Measurement of complex permittivity of Liquids Using waveguide Techniques. Progress in Electromagnetic Research, PIR, 2003, 4(2): 132-142.

[18] M.G. Corfield, J Horzelski, Rapid method for determining V.H.F. dielectric parameters for liquids and solutions using standing wave procedures, British Journal of
Applied Physics, 1961, 12(12): 680-684.

[19] M.B.R Murthy, B.S Dayasagar, and R L Patil, Measurements on polar liquids at a microwave frequency evaluation of molecular parameters-a new method. Journal of Physics, 2003, 18(4): 725-727.

[20] A.H. Abdul Jaleel, A.R. Khan, M. Faroqui, et al., Measurement of Dielectric Properties of Liquids Using Microwave Reflectometry Technique. IJIRSET, 2017, 6(8): 15915-15919.

[21] T. Hengcharoena, K. Eaiprasertsaka, and M. Fuangfoonga, Microwave Dielectric Measurement of Liquids by using Waveguide Plunger Technique, Proceedings of $2^{\text {nd }}$ International Science, Social Science, Engineering and Energy Conference 2010: Engineering Science and Management. Nakhonphanom, Thailand, Dec. 15-16, 2010: $270-274$

[22] P. R. Hiremath, Impact of Viscosity on Dielectric Relaxation Time of Some Organic Molecules. J. Adv. Phys. 2015, 4(2): 1-5.

[23] M.L. Sisodia, Basic Microwave Techniques and Laboratory Manual. New age publishers, India, 2005: 236-241.

[24] U.R. Lahane, Temperature Dependent Dielectric Relaxation Study of Arvindasava and Pipplyasava (Ayurvedic Medicines) in Ethanol. International Journal of Scientific \& Engineering Research, 2013, 4(5): 562565.

[25] U.R. Lahane, Dielectric parameter of ayurvedic medicines biological approach. IJRET, 2014, 3(1): 266-267.

[26] N. Kumar, D.K. Sinha, Drinking water quality management through correlation studies among various physicochemical parameters: A case study. IJES, 2010, 1(2): 253-259.

[27] A. Lothar, Correlation Coefficients: Appropriate Use and Interpretation. Nesthesia \& Analgesia, 2018, 126((5): $1763-1768$

[28] X. Li, Z. Chen, Y. Gao, et al., Probing the Debye dielectric relaxation in supercooled methanol. Frontiers in Materials, 2015, 2(4): 1-4.

[29] X. Tang, D. Fan, F. Hang, et al., Effect of Microwave Heating on the Dielectric properties and Components of Iron-Fortified Milk. Hindawi, Journal of Food Quality, 2017, 20(1): 1-10.

[30] Y. Liu, M. Yang, Y. Gao, et al., Broadband dielectric properties of honey: effects of temperature. J Food Sci Technol, 2019, 1: 34-38.

[31] M.K. Rendale, S.N. Mathad, Impact of Viscosity on Dielectric Relaxation Time of Some Organic Molecules. $P$. R. Journal of Advanced Physics, 2015, 4(2): 134-138.

Copyright $\subseteq$ Jinan Fadhil Mahdi, Ansari Abdul Jaleel Abdul Haque, Mazahar Ahmed Nazeeruddin Farooqui, and Yusuf Hanif Shaikh. This is an open-access article distributed under the terms of the Creative Commons Attribution License, which permits unrestricted use, distribution, and reproduction in any medium, provided the original author and source are credited. 